\title{
Main Barriers of the Implementation of the Blockchain Technology
}

\author{
Jan Zadrożny*
}

\begin{abstract}
Summary
Blockchain is a decentralized and distributed database, where participants can exchange the value of, i.e. information or financial resources without the participation of a trusted third party. Despite of a number of positive features, this technology is not free of defects. The aim of the article is to present the barriers of the discussed technology, which prevent the discussed technology from further dissemination. Therefore, in a first step, the review of basic concepts related to blockchain was conducted. It includes a discussion of key characteristics as well as dominant types of blockchain. Afterwards, the author presents barriers of the implementation of the blockchain technology: technical, socio-organizational and legal. Blockchain gained its popularity thanks to the bitcoin cryptocurrency, which was launched in 2009 as a consequence of the global financial crisis. Since then, the technology has gone a long way of evolution and development, and its use goes far beyond only the financial sector. Therefore, the article is not focused on the strengths and weaknesses of the bitcoin blockchain network, as this has already been the subject of many other publications (i.e. Yli-Huumo et al., 2016; Koteska et al., 2017), but aims to highlight barriers of its deployment. Consequently, the limitations faced by bitcoin blockchain or, more broadly, the world of cryptocurrencies, have been passed in this article advisedly.
\end{abstract}

Keywords: Blockchain technology, Distributed ledger, Limitations, Barriers, Innovation, BCT

JEL: O31, O33

\section{INTRODUCTION}

Blockchain does not belong to a technology that is easy to understand (Holotescu, 2018). This is due to the fact that it is associated with using an advanced cryptography. Despite of the early stage of development of the discussed technology, the community focused on the blockchain technology has created its own specialized language. Said language may pose difficulties for people who are just in the initial stage of starting their adventure with it. This all leads to a misunderstanding of the BCT concept, which can be exemplified by using the virtual bitcoin currency as a synonym of blockchain. While bitcoin is a cryptocurrency that works by using this technology, however, the blockchain also works without using Bitcoin. Having above in mind, in order to conduct an analysis of barriers, a basic review of the concepts is needed to understand the principles of the discussed problem.

Conceptual principles of operation of blockchain technology go back to 2009, when a person or group of people under the pseudonym of Satoshi Nakamoto issued a breakthrough article about the concept of a payment system. Said payment system in its assumptions aimed to enable the transaction of two entities directly to each other (P2P), without the participation or involvement of financial institutions (Nakamoto, 2008). The described methodology based on the bitcoin cryptocurrency was launched in 2009 and as it turned out - it caused a whole avalanche of events that led to the emergence and dynamic development of the entire ecosystem of blockchain technology extending far beyond the financial sector. Currently, the application of blockchain technology is observed in various sectors of the economy, i.e. logistics and supply chain (Francisco and Swanson, 2018), insurance, health care or the Internet of Things (IOT) (Khan and Salah, 2017), and even in state administration. A model example of implementation in the public sphere may be seen in Estonia, which, thanks to the use of blockchain, enables its citizens to vote electronically (Piech and Zyga,

* Faculty of Management, University of Warsaw, Poland, orcid.org/0000-0002-9796-808X, e-mail: zadrozny.jan@gmail. com 
2018). In the local government council elections in 2017 , nearly $32 \%$ of all voters decided to take advantage of e-voting (Estonian National Electoral Committee, 2017). This proves the legitimacy of implementing such a system, and may also be a remedy for relatively low voter turnout in our country (Korycki, 2017).

The given analysis carries the hallmarks of literature studies along with the use of deductive reasoning, according to the principles of the art of methodological correctness which strive to combine different techniques that happen to be complementary to each other (Apanowicz, 2002). Additionally, interviews and direct conversations with blockchain practitioners were also conducted. At the beginning of the article, the elementary concepts related to the discussed problem were explained simultaneously with the indication of the most important features of the technology. In the next step, on the basis of the literature review, the characteristics of the network types were made, with particular emphasis on the differences between individual functionalities. The central point of the work, which is also a contribution of the paper, is the presentation of barriers limiting the implementation of blockchain technology. The entire study closed by the summary.

\section{PROBLEM OF BYZANTINE GENERALS}

Blockchain technology solves the problem of Byzantine fault (Zheng et al., 2017), which previously was not possible with the help of well-known IT programs. The problem is built around an imaginary thought structure according to which the Byzantine army surrounds the city, and the general stands in front of the decision to attack or withdraw the army. The information is about to be given to the other generals by the messenger. If the assault is carried out at the right time, the conquest of the city will be possible. However, the difficulty can emerge if some of the generals turn out to be traitors who will intentionally pass a false message. Therefore, there is a need of creating a mechanism of action that will allow for the transmission of a proper message, even if a traitor participates in it. The solution to this problem is proposed by the blockchain technology, thanks to the use of the consensus.

\section{CONSENSUS}

The previous methods of confirming the occurrence of the situation referred to the establishing of a trusted third party that acted as an impartial body. The consensus mechanism eliminates this need, as the approval and joining of new blocks to the chain is performed by the blockchain network nodes. Therefore, the consensus can be defined as a mechanism of the agreement between nodes, thanks to which it is possible to add further information to the chain. Various types of algorithms can be used to achieve a consensus, such as (Bashir, 2017):

(1) The Nakamoto consensus, commonly used in the world of cryptocurrencies. It is a protocol that is used by the most popular cryptocurrencies such as bitcoin or ethereum that use proof of work (POW).

(2) The tolerance-based Byzantine fault - it is focused on performing voting rounds by nodes, which after reaching them reach a consensus. These types of protocols are most often used in private networks.

\section{KEY FEATURES OF BLOCKCHAIN}

This means that blockchain can be defined as a database where participants can exchange value (i.e. information, money) without the participation of a trusted third party. Therefore, initially blockchain was used in the world of cryptocurrencies, where members of the community made 
transactions directly among themselves. As rightly noted by A. Antonopoulous, there has been a shift from trusting people to trusting math (Antonopoulos, 2014). In this way, no institution in the world is responsible for such a constituted, public database. It is worth mentioning that the blockchain uses an advanced cryptography, resistant to hacking attacks and forgery.

The most important features of blockchain technology are presented in Table 1.

Table 1. Key characteristics of blockchain

\begin{tabular}{|c|c|}
\hline Features & Characterictics \\
\hline decentralization & $\begin{array}{l}\text { The block chain can be copied by any node in the network, which causes its } \\
\text { decentralization. Blockchain does not belong to any central infrastructure, therefore it will } \\
\text { work in the event of a breakdown or hacking attack. }\end{array}$ \\
\hline transparency & $\begin{array}{l}\text { Members have access to information contained in the block as well as in its entire } \\
\text { history. }\end{array}$ \\
\hline data security & Chain blocks are saved using advanced cryptography, so that stored data is secure. \\
\hline $\begin{array}{l}\text { operation based on } \\
\text { consensus }\end{array}$ & $\begin{array}{l}\text { Adding a block of information to the chain is possible when all relevant entities confirm } \\
\text { the correctness of the information. }\end{array}$ \\
\hline $\begin{array}{l}\text { chronological and timely } \\
\text { recording of data blocks }\end{array}$ & $\begin{array}{l}\text { The added blocks are marked with time, thanks to which they remain chronological in the } \\
\text { timeline. This allows for tracking the information chain without central records. }\end{array}$ \\
\hline durability & It is practically impossible to revoke the accepted information. \\
\hline digital record & $\begin{array}{l}\text { The information contained in the blockchain is digitized, which means that there is no } \\
\text { need to use the paper version. }\end{array}$ \\
\hline $\begin{array}{l}\text { reduction of third party } \\
\text { participation }\end{array}$ & $\begin{array}{l}\text { Based on the peer-to-peer principle of P2P. Network members interact with each other } \\
\text { without the intermediation of third parties, i.e. a central server. }\end{array}$ \\
\hline near real time & Blockchain allows you to settle and add new information almost in real time. \\
\hline
\end{tabular}

Table based on: Berryhill et al., 2018

\section{TYPES OF BLOCKCHAIN}

Blockchain technology was created as a result of the financial crisis, as an alternative to the prevailing financial system that did not work out (Biedrzycki, 2017). Therefore, it was initially used only by the bitcoin cryptocurrency, available to all users of the network. Cryptographic encryption and consensus protocol ensure that the information in the chain is reliable. Anyone who has access to the internet can become a member of the community and decide on the future of cryptocurrencies. Since that moment, other solutions have been created, which evolved with the development of the entire ecosystem. There are three main types of blockchain: public, private and consortium (Buterin, 2015). This division is extremely important because its choice determines the level of access and functionality.

\section{Public blockchain}

Access to the source code is open, which means that all network users can obtain access to it. Thanks to this, possible security imperfections can be identified and corrected by the community. In addition, the network is anonymous, decentralized and irrevocable, so it is not possible to make changes to the chain or censor it. This is a very useful feature, especially when network participants do not trust each other. Examples of networks implementing this model are i.e. ethereum or bitcoin. 
Therefore, the virtual currency bitcoin does not have a central authority responsible for its issue, authorization or approval of transactions.

The problem of public blockchains lays in their low efficiency due to high network maintenance costs. It results directly from the consensus based on the proof of work protocol (POW), where the participant of the network provides the computing power of his computer. The algorithm performs a number of mathematical calculations, the aim of which is to find the right sequence of numbers and verify its correctness, which results in adding another block to the chain. Network users compete for that who will be first, because the winner receives a bonus for the created block. To increase their chances, miners ${ }^{1}$ join in groups on the so-called pools. With the increase in the number of miners, the difficulty of extraction increases, which means that the amount of consumed electricity increases (Zambrano, 2017).

According to the Digiconomist's Bitcoin Energy Consumption Index, currently 2 the annual electricity consumption needed to maintain the bitcoin network is $73.12 \mathrm{TWh}$, which corresponds to $0.33 \%$ of the total world energy consumption. By comparison, it is more or less the same as Austria consumes annually (De Vries, 2018). In return, we receive a highly decentralized network, as well as a high level of security thanks to the enormous support of global computing power. In addition, public networks in commercial applications face numerous problems, i.e. (1) data privacy (2) ease of protocol update (3) data responsiveness (Hamida, Brousmiche, Levard i Thea, 2017). Although the lack of central management has its many advantages, group decision-making requires time that is needed to develop a common position. Therefore, other solutions have been created: private blockchain and consortium blockchain, whose main distinguishing feature is the network management criterion.

\section{Private blockchain}

Network anonymity is not always a desirable feature. In the course of business, the customer would like to know who the supplier is and who the recipient of the goods and services are. Scalability 3 is also very important. Therefore, to meet the market's challenges, a private blockchain was created, which - as the name suggests - is controlled by the organization. In this model, the administrator (owner of the network) arbitrarily assigns the rights to individual users and assigns responsibility to them so that they can be modified at the same time. This solution increases control over the flow of values in the chain, which means that one can give up the costly consensus protocol based on proof-of-work. On the other hand, it contributes to greater vulnerability to hacking attacks.

\section{Consortium blockchain}

While in a public blockchain every human being can become part of a network, here only a group of nodes can participate in the consensus protocol. This means that although the selected group controls the consensus process, other nodes may be able to participate by obtaining special permissions, i. e. viewing the history of the chain or creating new blocks. Consortium blockchains are faster and provide greater transaction privacy (or information). This type of network is used by the banking sector (Rice, 2017).

Table 2 summarizes the previous considerations of the types of the chains. It is worth analyzing them, because each of them has its advantages and disadvantages as well as limitations.

Defined as participants of the loosely organized network

As of August 2018

Defined as the ability of the network to process a certain number of transactions per second 
Table 2. Comparison of public, private and consortium blockchain network type

\begin{tabular}{llll}
\hline & \multicolumn{1}{c}{ Public blockchain } & Private blockchain & Consortium blockchain \\
\hline Consensus determination & all miners & one organization & selected group of nodes \\
Level of centralization & fully decentralized & fully centralized & quasi-centralized \\
Read permisssion & public & public or restricted & public or restricted \\
Cost of keeping consensus & highest & the lowest & lower \\
Security level & highest & the lowest & lower \\
\hline
\end{tabular}

Table based on: Zheng, et al., 2017

Figure 1 graphically illustrates the differences between the types of given networks. Black color means the level of access to information, source code and functionality, while elliptical figures symbolically represent network users. Their size does not matter. According to the figure, in a public blockchain every network user has full access to information. In a private blockchain there is one node that has full access to information, while other network participants have limited access to it. In the third type, the access to information has several nodes, in this drawing specifically two.

Figure 1. Graphical presentation of blockchain network types. Black points mean nodes, while white points are network users

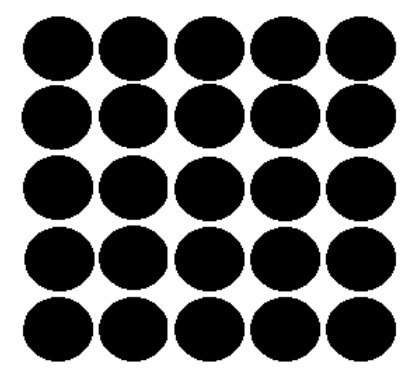

Publik blockchain

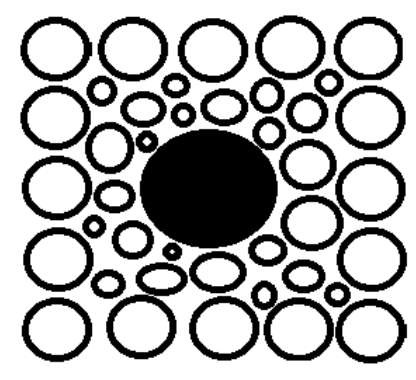

Private blockchain

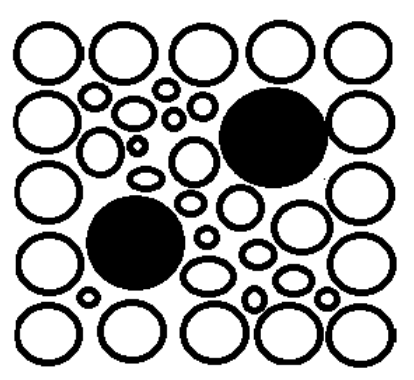

Consortium blockchain

Sources: own elaboration

The selection of the block's network type depends on the purpose for which it is to be used. For example, if we care about full transparency and decentralization of the database, then the solution offered by the public network should be used. Therefore, the choice of the type of network is a key decision determining the later functionalities of the system.

\section{KEY LIMITATIONS OF BLOCKCHAIN}

Blockchain solves the problem of safe and reliable transfer of value (i.e. information, money) between two equal entities without the necessity of participation of a trusted third party, which is why it observes its use in industry. As a new field, it has a number of barriers that may delay or stop its further popularization. It is possible to classify the limitations on the following factors: (1) technical, i.e. those that result directly from the weakness of the technology. This category should include issues related to the complexity, irrevocability, as well as the costs of network 
service; (2) socio-organizational concern of human activity, which is why the main barriers are: lack of infrastructure, demolition of the current order, and a small number of implementations; (3) legal that related to the need for regulation changes.

\section{Technological barriers of the given technology}

Complexity. Blockchain uses advanced cryptography, which makes it a complicated technology, difficult to understand also at the programming level. In addition, BCT, despite its young age, introduced its own terminology to the IT industry. The language used may be difficult to understand for people who are just joining the blockchain community. It requires IT specialists to learn new programming languages, i.e. Solidity. Courses and trainings have recently appeared on the market, which is why today we can talk about the lack of specialists in this field.

Network immutability. This feature is one of the most important benefits of using blockchain, which paradoxically can become a problem in the case of further dissemination of Blockchain technology. It allows you to add further blocks of information without the possibility of their subsequent removal. What if the sensitive information is added to the block, i.e. concerning the employment of employees? An employer, depending on the jurisdiction of a given country, has the obligation to archive data, but it has a temporary character, not an infinite one. Consider whether it is appropriate to use this technology if you edit and delete information from the database frequently.

Service costs. Although in the root assumptions the technology was supposed to eliminate high transaction costs, in practice it may cause for them to increase. In addition to the previously described, economically inefficient, consensus protocol based on the principle of POW (proof-ofwork), it is worth completing the issue of the irrevocability (network immutability) of the network. In addition to the inability to edit content in the chain, the data is stored indefinitely, which entails significant maintenance costs for each decentralized network, where the size of stored data will constantly increase.

\section{Social and organizational barriers}

Lack of infrastructure. The immense potential of blockchain technology lies in its global use, i.e. logistics or supply chain. Third world countries, or developing countries, are not prepared for this technology due to the low level of digitization and technical infrastructure.

Demolishing the current order - the introduction of blockchain technology on a global scale will eliminate the presence of some middlemen. Going further, its implementation will change many relationships and business models, where international relationships built on trust will cease to play a role. It is expected that this will result in opposition of environments that will lose on its implementation. In addition, resistance to change is an immanent feature of the organization and accompanies employees who, in fear of change, strive to maintain their current status quo. As noted by M. Centkowska, employees most strongly oppose the changes that make their existing skills insufficient (Centkowska, 2015). Therefore, this factor will act as a brake on further dissemination of technology.

A small number of implementations - another obstacle that hinders the further development of the industry is a small number of implementations. Business owners will start making decisions on using technologies on a mass scale when they are convinced of its benefits.

\section{The legislative barrier}

Legislative restrictions concern the lack of appropriate legal regulations. Innovations and new technologies usually precede the existing legal order. Therefore, the key element is the flexibility and attitude of the regulations to the dynamically changing market situation. The friendly 
attitude of the authorities to the newly emerging initiatives and technological solutions based on blockchain, such as a smart contract, is fundamental for the further development of the industry. Lack of case law introduces uncertainty, which means that organizations will not be willing to invest and use technologies until the introduction of additional regulations in the discussed area will be completed.

\section{CONCLUSIONS}

Blockchain represents a different approach of the collecting, processing and data analysis, which will contribute to the transformation of existing solutions and will affect the change of business models. The technology is at an early stage of development, which means that more implementations as well as time are needed. This will allow us to assess, understand and learn about other, unknown technological barriers that may slow down the process of further adaptation. The support from the state seems to be necessary, especially in order to encourage research centres and enterprises to conduct further research in this area.

\section{Literature}

Antonopoulos, A. (2014). Bitcoin Security Model: Trust by Computation. O'Reilly-Radar. Retrieved from http:// radar.oreilly.com/2014/02/bitcoin-security-model-trust-by-computation.html (28.08.2018).

Apanowicz, J. (2002). Metodologia ogólna, Wydawnictwo Diecezji Peplińskiej „BERNARDINUM”.

Bashir I. (2017). Mastering Blockchain: Distributed ledgers, decentralization and smart contracts explained, Published by Packt Publishing, Birmingham, UK.

Biedrzycki, N. (2017). Blockchain- the ultimate financial crash. Retrieved from https://norbertbiedrzycki.pl/en/ blockchain-czyli-ostateczny-krach-systemu-finansowego/ (18.08.2018).

Berryhill, J., Bourgery T., Hanson, A. (2018), Blockchains Unchained: Blockchain Technology and its Use in the Public Sector, OECD Working Papers on Public Governance, No. 28, OECD Publishing, Paris.

Buterin, V. (2015). On public and private blockchains. Retrieved from https://blog.ethereum.org/2015/08/07/ on-public-and-private-blockchains/ (19.082018).

Centkowska, M. (2015). Podstawy teoretyczne oporu wobec zmian w organizacji, Zeszyty Naukowe Politechniki Śląskiej, seria: „Organizacja i Zarządzanie”, 77, 9-18.

De Vries, A. (2018). Bitocin's Growin Energy Problem, Joule, 2. 801-809.

Estonian National Electoral Committee (2017). Retrieved from https://kov2017.valimised.ee/valimistulemus-vald.html (22.08.2018).

Francisco, K., Swanson, D. (2018). The supply chain has no clothes: technology adoption of Blockchain for supply chain transparency, Logistics 2018, 2, 2 https://doi.org/10.3390/logistics2010002.

Hamida, E.B., Brousmiche, K. L., Levard, H., Thea, E. (2017). Blockchain for Enterprise: Overview, Opportunities and Challenges. in The Thirteenth International Conference on Wireless and Mobile Communications (ICWMC 2017), Nice, France, Jul. 2017.

Holotescu, C. (2018). Understanding technology and how to get involved, in the he 14th International Scientific Conference eLearning and Software for Education Bucharest, April 19-20, http://dx.doi.org/10.12753/2066$-026 \mathrm{X}-18-253$.

Khan, M.A., Salah, K.(2017). IoT security: review, blockchain solutions, and open challenges, In: Future Generation Computer Systems. http://dx.doi.org/10.1016/j.future.2017.11.022.

Korycki, K. (2017). Alternatywne techniki głosowania a frekwencja wyborcza. Studia wyborcze, 23, 83-105.

Koteska, B., Karafiloski, E. Mishev, A. (2017). Blockchain Implementation Quality Challenges: A Literature Review. In Proceedings of SQAMIA 2017: 6th Workshop of Software Quality, Analysis, Monitoring, Improvement, and Applications. Belgrade, Serbia, September 11-13. Retrieved from http://ceur-ws.org/Vol-1938/ paper-kot.pdf (31.08.2018).

Nakamoto, S. (2008). Bitcoin: A peer-to-peer electronic cash system. Retrieved from https://bitcoin.org/bitcoin. pdf (20.08.2018).

Piech, K., Zyga, P.(2018). Wykorzystanie blockchain przez rząd estoński. Retrieved from https://www.lazarski. pl/pl/wydzialy-i-jednostki/instytuty/wydzial-ekonomii-i-zarzadzania/centrum-technologii-blockchain/wykorzystanie-blockchain-przez-rzad-estonski/ (22.08 2018). 
Rice, A.D. (2017), Blockchain: A Beginner's Guide to Blockchain Technology, CreateSpace Independent Publishing Platform.

Yli-Huumo, J., Ko, D., Choi, S., Park, S., Smolander, K. (2016). Where is current research on blockchain technology? - A systematic review. PLoS One, 11(10), https://doi.org/10.1371/journal.pone.0163477.

Zambrano, R.(2017). Blockchain: Unpacking the disruptive potential of blockchain technology for human development, International Development Research Centre. Retrieved from https://idl-bnc-idrc.dspacedirect.org/ bitstream/handle/10625/56662/IDL-56662.pdf?sequence=2\&isAllowed=y (31.08. 2018).

Zheng, Z., Xie, S., Dai, H., Chen, X., Wang, H. (2017). An overview of Blockchain Technology: architecture, consensus, and future trends. In: IEEE 6th International Congress on Big Data. http://dx.doi.org/10.1109/ BigDataCongress.2017.85 